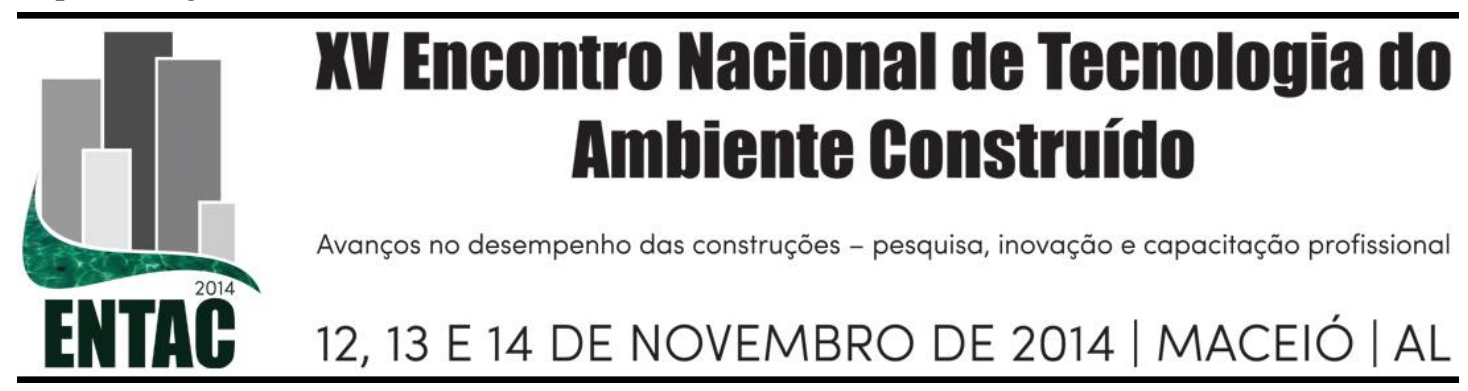

\title{
REGRESSÃO LOGÍSTICA E GRAUS-HORA NA AVALIAÇÃO DO DESEMPENHO TÉRMICO DA ENVOLTÓRIA
}

\author{
OLIVEIRA, Andréia (1); LEDER, Solange (2); GOULART, Solange (3)
}

(1) Universidade Federal da Paraíba, e-mail: andreiaoliveira.arq@ gmail.com (2) Universidade Federal da Paraíba, e-mail: solangeleder@yahoo.com.br, (3) Universidade Federal do Rio Grande do Norte, e-mail: aasolange@hotmail.com

\begin{abstract}
RESUMO
Este trabalho tem como objetivo avaliar o desempenho térmico de fechamentos opacos em edifícios ventilados naturalmente utilizando o TRY para predição da temperatura operativa. $O$ estudo foi desenvolvido com a definição de modelos de simulação para o município de Patos - Paraíba, com método dividido em três etapas: 1) caracterização do clima; 2) caracterização dos modelos e 3) avaliação de desempenho térmico. Foram comparados dois métodos de avaliação: Graus-hora para resfriamento e Regressão Logística. Pôde-se observar que o uso de materiais com isolamento térmico nos fechamentos opacos horizontais reduziu a chance de conforto. Por outro lado, o isolamento resultou em condições menos desconfortáveis no interior da edificação, nos horários mais quentes, por reduzir as oscilações térmicas do ambiente, mantendo a temperatura operativa com pequenos intervalos entre o dia e a noite, embora com temperaturas acima do limite de conforto. Diferentes interpretações das condições de conforto podem ocorrer, dependendo do método de análise utilizado.
\end{abstract}

Palavras-chave: Desempenho térmico, Conforto, Fechamentos Opacos.

\begin{abstract}
This paper aims to evaluate the thermal performance of opaque closures naturally ventilated buildings using the TRY to predict operative temperature. The study was developed with the definition of simulation models for the city of Patos - Paraíba. The method was divided into three stages: 1 ) climate characterization; 2 ) models characterization and 3 ) evaluation of thermal performance. Two evaluation methods were compared: Degree hour for cooling and Logistic regression. It was observed that the use of materials with thermal insulation in the horizontal opaque closures reduced the chance of comfort. On the other hand, isolation resulted in less uncomfortable conditions inside the building, in the hottest times by reducing the thermal fluctuations of the environment while maintaining the operating temperature with small intervals between day and night, although temperatures above the comfort limit. Differing interpretations of comfort conditions may occur depending on the method of analysis used.
\end{abstract}

Keywords: Thermal performance, Comfort, Opaque closures.

\section{INTRODUÇÃO}

Estudos relacionados à eficiência energética e a redução do consumo de energia tem utilizado diversos índices de conforto como parâmetros para a avaliação de desempenho térmico das edificações. Considerando que o estado de conforto térmico resulta das trocas térmicas entre o homem e o ambiente, e que mudanças térmicas do exterior refletem diretamente em edifícios ventilados naturalmente, percebe-se a importância de entender o comportamento térmico da envoltória. 
Diversos autores desenvolveram pesquisas sobre os índices de avaliação de conforto térmico, tais como Aste, Angelotti e Buzzet (2009), Barrios et al. (2012), Carlucci e Pagliano (2012), Gasparella et al. (2011). Segundo esses, os índices de conforto não expressam todas as condições de conforto encontradas no edifício, devendo-se ter cuidado na escolha e na maneira de usá-los. Barrios et al. (2012) comentam que nem todos os índices de conforto são adequados para edifícios com ventilação natural.

Este trabalho tem como objetivo avaliar o desempenho térmico de fechamentos opacos em edifícios ventilados naturalmente utilizando o TRY para predição da temperatura operativa. O estudo foi desenvolvido com a definição de modelos de simulação de uma edificação ventilada naturalmente com variações de fechamentos opacos horizontais e verticais, para o clima de Patos - Paraíba. Foram comparados dois métodos de avaliação: o método de Graus-hora para resfriamento e o método de Regressão Logística, a partir da temperatura operativa, com o objetivo de verificar a hipótese de que métodos estatísticos podem auxiliar no entendimento do desempenho térmico da envoltória das edificações como forma de detalhar a análise das condições dinâmicas em ambientes com maior variabilidade climática.

\section{REVISÃO BIBLIOGRÁFICA}

Estudos sobre conforto térmico observaram que pessoas que vivem ou trabalham em edifícios naturalmente ventilados, com controle de aberturas, demonstraram uma maior adaptação à variabilidade climática sazonal e a diversidade de temperaturas diárias, resultando em uma tolerância maior às temperaturas acima da zona de conforto adotada. Baseado neste comportamento, os modelos adaptativos propõem um ajuste ao padrão de conforto térmico sob condições dinâmicas, que considera o uso de temperaturas horárias, mais apropriado que médias mensais para utilização de predição de temperaturas de conforto (ASHRAE, 2010; NICOL e HUMPHREYS, 2002).

Segundo Nicol e Humphreys (2002), o modelo adaptativo fundamenta-se no princípio que "se ocorre uma mudança de modo a produzir desconforto, as pessoas reagem de modo que tendem a restaurar sua condição de conforto". Desta maneira, este modelo aborda a possibilidade das pessoas se adaptarem às mudanças térmicas ocorridas em seus ambientes, modificando vestimentas, alterando dispositivos de ventilação, etc.

O conceito de temperatura operativa, segundo a norma 15.220 (ABNT, 2005) descreve que é a temperatura uniforme de um ambiente com comportamento de corpo negro imaginário no qual o ocupante poderia trocar a mesma quantidade de calor por radiação e convecção que no ambiente real não uniforme. A temperatura operativa pode ser obtida a partir da equação abaixo, como resultado da ponderação entre a temperatura do ar e a temperatura radiante média do ambiente (ASHRAE, 2010).

$$
T o=\left(\frac{T a+T r}{2}\right)
$$

Onde:

To $=$ temperatura Operativa

$\mathrm{Ta}=$ temperatura do ar

$\operatorname{Tr}=$ temperatura média radiante

\subsection{Graus-hora para Resfriamento}

Graus-dia e Graus-hora são métodos desenvolvidos pela ASHRAE para cálculo de consumo de energia, sendo um dos métodos de avaliação de desempenho mais utilizado 
atualmente. O Graus-hora é calculado tomando-se como base a temperatura de conforto máxima (para resfriamento) e mínima (para aquecimento). Este método permite a observação do excedente da temperatura em relação a uma temperatura base, que varia de acordo com a temperatura externa.

$$
\mathrm{GhR}=\sum(\mathrm{Tb}-\mathrm{To})>0
$$

onde, GhR = Graus-hora para resfriamento

$\mathrm{Tb}=$ Temperatura base

To = Temperatura operativa

O valor resultante desta equação é utilizado para aquecimento, ou seja, quando a temperatura operativa horária (To) for menor que $\mathrm{Tb}$ (temperatura base), calcula-se a diferença, somando-se os valores para todo o ano. Da mesma maneira, quando ela for maior que $\mathrm{Tb}$, o resultado desta soma é utilizado para cálculo de resfriamento (BARRIOS et al., 2012; GOULART, 1993).

\subsection{Regressão Logística}

A Regressão Logística é um método estatístico da classe dos modelos lineares generalizados. Os modelos lineares generalizados são modelos estatísticos compostos apenas por parâmetros e variáveis, também chamados de modelos paramétricos. São obtidos a partir de um algoritmo geral para computação de estimativas de máxima verossimilhança, permitindo o ajuste de diferentes modelos para diferentes tipos de escala, quantitativas ou qualitativas (CORDEIRO e ANDRADE, 2009; CORDEIRO e DEMÉTRIO, 2008).

O uso da Regressão Logística permite quantificar a diferença entre combinações, considerando uma variável binomial. Este modelo considera uma variável resposta binária, identificada por $\mathrm{Yi}$, baseada nos valores, $\mathrm{Yi}=0$ e $\mathrm{Yi}=1$, denominados "fracasso" e "sucesso", respectivamente, considerando o "sucesso" como evento de interesse. Para Hosmer e Lemeshow (2000) um coeficiente importante quando se trabalha com regressão logística é a odds ratio (razão da chance), que informa o efeito da variação em uma determinada variável sobre a chance de ocorrência de um evento. Para uma variável $X_{j}$ a razão da chance é expressa pela equação 3 .

$$
\psi=\exp \left(\beta_{j}\right)
$$

No modelo de regressão logística, a equação resultante indica que o coeficiente de cada variável independente aumenta em função da chance do evento ocorrer pela variável resposta, conforme equação abaixo.

$$
\text { chance }=\frac{\mu}{1-\mu}=e^{\beta_{0}+\beta_{1} X_{1}+\ldots+\beta_{k} X_{k}}=e^{\beta_{0}} \cdot\left(e^{\beta_{1}}\right)^{X_{1}} \ldots\left(e^{\beta_{k}}\right)^{X_{k}}
$$

Para diagnosticar as irregularidades do modelo, foram aplicadas duas análises de resíduos. O teste da razão de verossimilhança, que indica o nível de associação entre as variáveis independentes e a variável resposta, e o teste qui-quadrado de Pearson, aplicado para verificar a independência de duas variáveis qualitativas (DEVORE, 2006). 


\section{MÉTODO}

\subsection{Caracterização climática}

O clima da cidade de Patos, pertencente a zona bioclimática 8 , foi analisado a partir do Ano Típico - TRY - desenvolvido pelo grupo da ANTAC e publicada no site do LABEEE-UFSC.

\subsection{Caracterização dos modelos}

O programa de simulação computacional escolhido para utilização neste trabalho foi o Design Builder, por ser um dos principais programas utilizados no Brasil e utilizar o formato (.EPW) de base de dados climáticos relativo a um ano típico de referência TRY, permitindo simulações horárias no período de um ano. O modelo base foi configurado em quatro abas distintas: padrão de ocupação, padrão de construção, padrão de abertura e padrão de consumo energético. As simulações foram realizadas com os padrões de ocupação, padrão de abertura e de consumo energético, fixos.

Os modelos simulados foram determinados como ambientes ventilados naturalmente, com a possibilidade de operação das aberturas que reproduzem os hábitos dos ocupantes, seguindo o padrão de ocupação, com acionamento da rotina (scheduler) para temperaturas do ar internas superiores a $26^{\circ} \mathrm{C}$. Em todos os casos foram desconsiderados o uso de sistemas artificiais de condicionamento, sendo desativado este setpoint no programa.

O consumo mensal de energia elétrica estimado foi de $150 \mathrm{kWh}$, considerando o consumo total dos equipamentos e do sistema de iluminação, a partir da descrição dos equipamentos presentes por unidade. Para a faixa de renda familiar de até 03 salários mínimos os equipamentos são: aparelho de som, chuveiro elétrico, ferro de passar, fogão, geladeira, liquidificador, televisão e ventilador (TAVARES, 2006).

Para o caso base, foi adotada uma unidade habitacional unifamiliar, como modelo representativo de habitação de interesse social, com $35 \mathrm{~m}^{2}$ de área total construída, com os seguintes ambientes: 1 sala, 1cozinha, 1 banheiro e 2 quartos. A edificação é térrea e possui a cobertura em duas águas no sentido leste/ oeste, conforme planta da figura 1:

\section{Figura 1 - Planta baixa e perspectiva do modelo base}
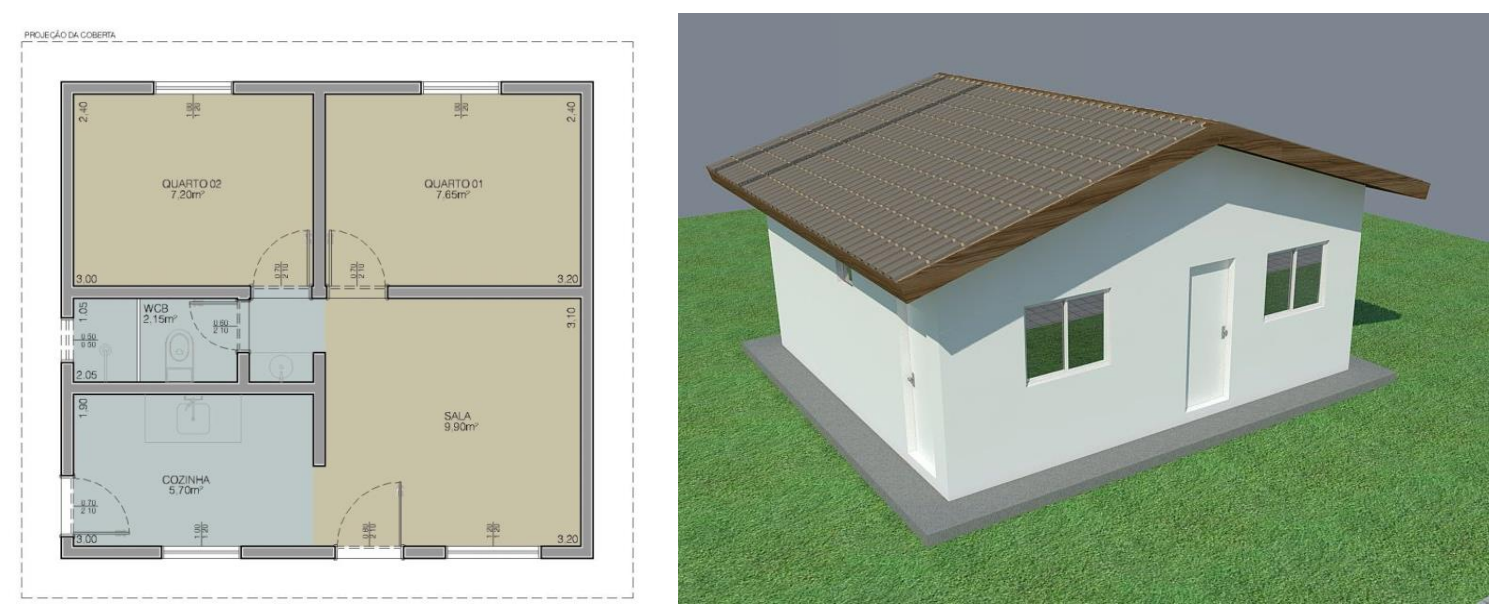

Fonte: Autor 
A partir das indicações de fechamentos verticais opacos sugeridos pela norma NBR 15.220 (ABNT, 2005), foram selecionadas 2 tipos de fechamento vertical opaco considerando o menor e maior valor de transmitância térmica e atraso térmico, indicados para todas as zonas bioclimáticas. Seguem abaixo, na tabela 1, os códigos e descrições dos tipos de fechamentos verticais opacos (parede) adotados para esta pesquisa, com os valores das seguintes propriedades térmicas: U (transmitância térmica), Ct (Capacidade térmica), $\alpha$ (absortância solar); $\Phi$ (atraso térmico).

Tabela 1 - Descrição das paredes

\begin{tabular}{c|c|c|c|c|c}
\hline Cod. & Descrição & $\begin{array}{c}\mathbf{U} \\
{\left[\mathbf{W} /\left(\mathbf{m}^{2} \cdot \mathbf{K}\right.\right.} \\
)]\end{array}$ & $\begin{array}{c}\mathbf{C t} \\
{\left[\mathbf{k J} /\left(\mathbf{m}^{2} \cdot \mathbf{K}\right.\right.} \\
)]\end{array}$ & $\boldsymbol{\alpha}$ & $\begin{array}{c}\mathbf{\Phi} \\
{[\mathbf{h o r a s}} \\
]\end{array}$ \\
\hline PA & $\begin{array}{c}\text { Parede de tijolos de 8 furos circulares, } \\
\text { assentados na menor dimensão. Espessura } \\
\text { total da parede: } 15,0 \mathrm{~cm}\end{array}$ & 2,24 & 167 & 0,20 & 3,7 \\
\hline PB & $\begin{array}{c}\text { Parede de concreto maciço } \\
\text { Espessura total da parede: } 5,0 \mathrm{~cm}\end{array}$ & 5,04 & 120 & 0,20 & 1,3 \\
\hline PC & $\begin{array}{c}\text { Parede dupla de tijolos de } 8 \text { furos } \\
\text { circulares, assentados na maior dimensão. } \\
\text { Espessura total da parede: } 46,0 \mathrm{~cm}\end{array}$ & 0,98 & 368 & 0,20 & 10,8 \\
\hline
\end{tabular}

Fonte: Adaptado de NBR 15.220 (ABNT, 2005)

Em seguida, foram selecionados 4 tipos de fechamentos horizontais opacos, considerando também o menor e maior valor de transmitância térmica e atraso térmico, indicados pela norma NBR 15.220 (ABNT, 2005). Seguem abaixo os códigos e descrições dos fechamentos horizontais opacos (cobertura) na tabela 2.

Tabela 2 - Descrição das coberturas

\begin{tabular}{|c|c|c|c|c|c|}
\hline Cod. & Descrição & $\begin{array}{c}\mathbf{U} \\
{\left[\mathbf{W} /\left(\mathbf{m}^{2} \cdot \mathbf{K}\right)\right]}\end{array}$ & $\begin{array}{c}\mathbf{C t} \\
{\left[\mathbf{k J} /\left(\mathbf{m}^{2} \cdot \mathbf{K}\right.\right.} \\
\quad)]\end{array}$ & $\boldsymbol{\alpha}$ & $\begin{array}{c}\phi \\
{[\text { horas }} \\
]\end{array}$ \\
\hline $\mathrm{CA}$ & $\begin{array}{l}\text { Cobertura de telha de barro com forro de } \\
\text { concreto. Espessura do concreto: } 3,0 \mathrm{~cm}\end{array}$ & 2,24 & 84 & 0,7 & 2,6 \\
\hline $\mathrm{CB}$ & $\begin{array}{c}\text { Cobertura de telha de barro com } 5,0 \mathrm{~cm} \text { de } \\
\text { lã de vidro sobre o forro de madeira. } \\
\text { Espessura da madeira: } 1,0 \mathrm{~cm}\end{array}$ & 0,62 & 34 & 0,7 & 3,1 \\
\hline $\mathrm{CC}$ & $\begin{array}{l}\text { Cobertura de telha de barro, lâmina de } \\
\text { alumínio polido e laje de concreto de } 25 \\
\mathrm{~cm} \text {. Espessura da telha: } 1,0 \mathrm{~cm}\end{array}$ & 1,03 & 561 & 0,7 & 13,4 \\
\hline $\mathrm{CD}$ & $\begin{array}{l}\text { Cobertura de telha de fibrocimento sem } \\
\text { forro. Espessura da telha: } 0,7 \mathrm{~cm}\end{array}$ & 4,60 & 18 & 0,7 & 0,2 \\
\hline
\end{tabular}

Fonte: Adaptado de NBR 15.220 (ABNT, 2005) 


\subsection{Avaliação de desempenho térmico}

A partir do conceito de modelo adaptativo definido por De Dear e Brager (2002), foram utilizados dados de temperatura operativa como índice de conforto térmico para a definição de Graus-hora para Resfriamento e para Regressão Logística.

No método de Graus-hora, foi somado o valor excedente da temperatura operativa que esteve acima da temperatura base de $26,5^{\circ} \mathrm{C}$, determinada de acordo com o gráfico de referência de temperatura operativa $\mathrm{x}$ temperatura externa, da norma Standard 55 (ASHRAE, 2010).

$$
\mathrm{GhR}=\sum(26,5-\mathrm{To})>0
$$

onde, $\mathrm{GhR}=$ Graus-hora para resfriamento

$\mathrm{Tb}=$ Temperatura base

To $=$ Temperatura operativa

O uso da Regressão Logística permitiu verificar a influência das combinações entre parede e coberta sobre o conforto térmico no interior do ambiente. No modelo de Regressão Logística, foram comparadas os dados horários de temperatura operativa das combinações, considerando como evento de interesse $(\mathrm{y}=1)$ os valores de temperatura operativa abaixo da temperatura base de $26,5^{\circ} \mathrm{C}$. A partir da equação 4 determinada para este modelo, foram determinados o melhor e o pior desempenho entre as combinações.

$$
\ln \left(\frac{p}{1-p}\right)=\beta_{o}+\beta_{1} \text {. Grupo }
$$

\section{RESULTADOS E DISCUSSÃO}

O clima considerado nesta pesquisa é referente à cidade de Patos na Paraíba. Com latitude de $7,02^{\circ}$ sul e longitude de $37,27^{\circ}$ oeste, a cidade está situada a $249 \mathrm{~m}$ de altitude, com umidade média de $62,04 \%$ e o nível pluviométrico anual de $1.603,20 \mathrm{~mm}$. As temperaturas médias mensais apresentam valores entre $25^{\circ} \mathrm{C}$ a $29^{\circ} \mathrm{C}$. As mínimas ocorrem entre os meses de junho, julho e agosto, chegando a $21^{\circ} \mathrm{C}$. As médias mensais das temperaturas máximas ocorreram entre $30^{\circ} \mathrm{C}$ e $36^{\circ} \mathrm{C}$, sendo outubro, novembro e dezembro os meses mais quentes, como pode ser observado no gráfico 1 .

\section{Gráfico 1 - Amplitude térmica em Patos}

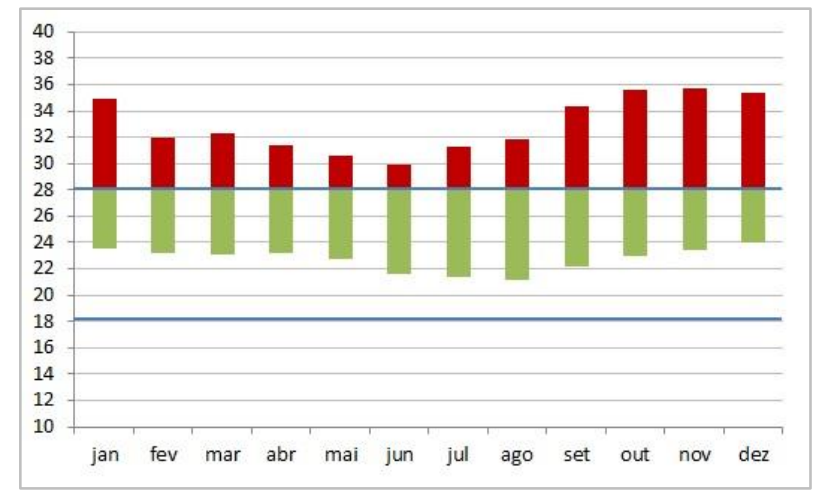

Fonte: Autor 
Observam-se valores de temperatura que causam desconforto por calor em todos os meses do ano. Destacam-se a mínima absoluta de $18,80^{\circ} \mathrm{C}$ em março, maio e julho, e a máxima absoluta de $37,70^{\circ} \mathrm{C}$ em janeiro, maio e novembro.

\subsection{Graus-hora para Resfriamento}

O método de análise de Graus-hora para resfriamento (GhR) demonstrou diferenças significativas entre as combinações. A maior diferença ocorreu na coberta (D), com telha de fibrocimento sem forro, que resultou nos valores mais altos, variando entre 37.053,97 GhR, quando combinada com a parede (B), e 39.534,53 GhR, na combinação com a parede $(\mathrm{C})$, como pode ser observado no gráfico 2 abaixo.

\section{Gráfico 2 - GhR dos fechamentos opacos em Patos}

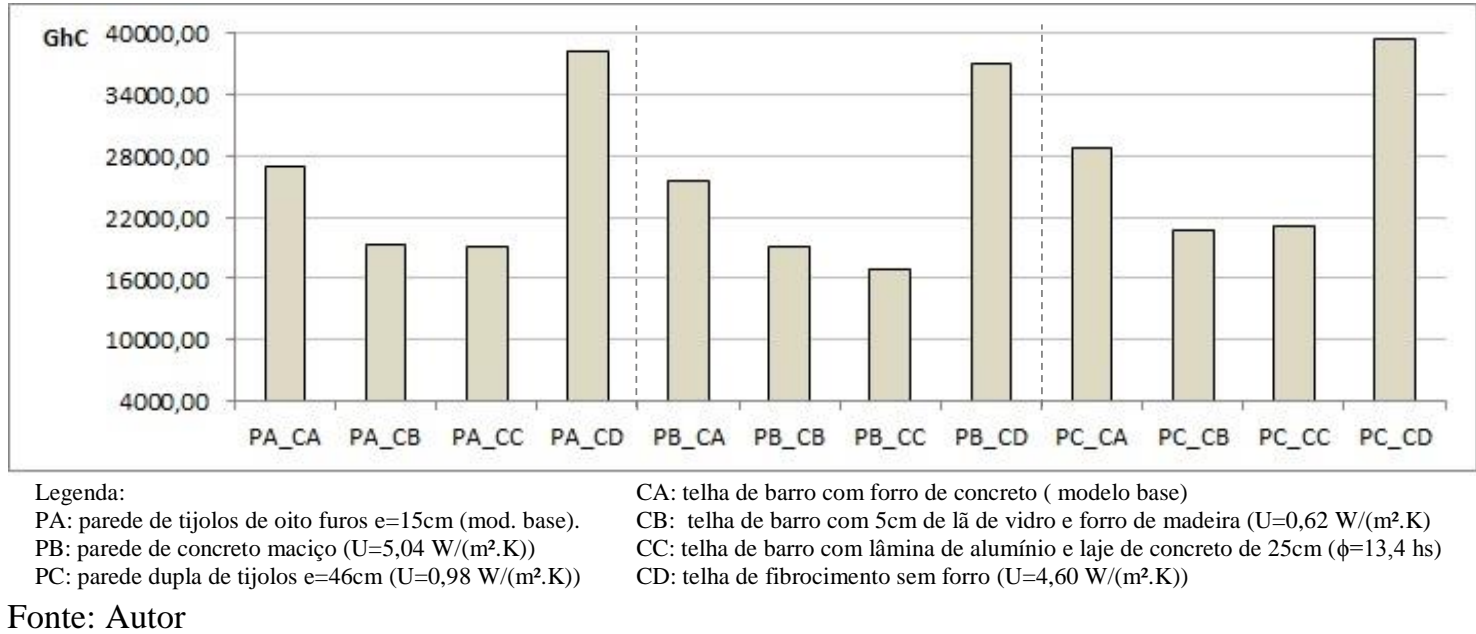

Pôde-se observar que as cobertas (B) e (C), que possuem isolamento térmico em sua composição, resultaram nos melhores desempenhos e com comportamento semelhante, quando combinadas com as paredes em análise. O melhor desempenho resultou da combinação da parede (B), de concreto maciço, com a coberta (C), de telha de barro, lâmina de alumínio e laje de concreto, que possui 16.871,46 GhR.

\subsection{Regressão Logística}

Inicialmente, foram determinadas as estimativas e os coeficientes de significância a partir do Teste de Wald, a fim de identificar as diferenças significativas entre as combinações, quando comparadas com o modelo base: parede (A) e a coberta (A). A partir da tabela 3, pôde-se observar que houve diferença significativa entre todas as combinações e o modelo base, rejeitando a hipótese nula. A partir das estimativas, foram calculados os exponenciais, que permitiram quantificar a razão da chance de ocorrer o evento de interesse e a diferença entre cada categoria simulada.

Tabela 3 - Coeficientes de significância To - Patos

\begin{tabular}{c|c|c|c|c|c}
\hline Coeficientes & Estimativas & $\operatorname{Pr}(>|\mathbf{z}|)$ & Resultado & Exponenciais & Chance \\
\hline (Intercept) & 0,047726 & 0,103 & Rejeita a Ho & 1,04 & \\
\hline factor(G)2 & 0,231325 & $4,29 \times 10^{-10}$ & Rejeita a Ho & 1,26 & Aumenta em \\
\hline
\end{tabular}




\begin{tabular}{|c|c|c|c|c|c|}
\hline & & & & & $26 \%$ \\
\hline factor $(\mathrm{G}) 3$ & $-0,713791$ & $<2 \times 10^{-16}$ & Rejeita a Ho & 0,49 & Reduz em 51\% \\
\hline factor $(\mathrm{G}) 4$ & 0,691750 & $<2 \times 10^{-16}$ & Rejeita a Ho & 1,99 & $\begin{array}{c}\text { Aumenta em } \\
99 \%\end{array}$ \\
\hline factor $(\mathrm{G}) 5$ & 0,508150 & $<2 \times 10^{-16}$ & Rejeita a Ho & 1,66 & $\begin{array}{c}\text { Aumenta em } \\
66 \%\end{array}$ \\
\hline factor $(\mathrm{G}) 6$ & 0,812173 & $<2 \times 10^{-16}$ & Rejeita a Ho & 2,25 & $\begin{array}{c}\text { Aumenta em } \\
125 \%\end{array}$ \\
\hline factor $(\mathrm{G}) 7$ & $-0,165723$ & $1,83 \times 10^{-5}$ & Rejeita a Ho & 0,85 & Reduz em 15\% \\
\hline factor $(G) 8$ & 0,990212 & $<2 \times 10^{-16}$ & Rejeita a Ho & 2,69 & $\begin{array}{c}\text { Aumenta em } \\
169 \%\end{array}$ \\
\hline factor $(\mathrm{G}) 9$ & $-0,359095$ & $<2 \times 10^{-16}$ & Rejeita a Ho & 0,69 & Reduz em $31 \%$ \\
\hline factor $(\mathrm{G}) 10$ & $-0,176627$ & $5,1 \times 10^{-6}$ & Rejeita a Ho & 0,83 & Reduz em 17\% \\
\hline factor(G)11 & $-1,247628$ & $<2 \times 10^{-16}$ & Rejeita a Ho & 0,29 & Reduz em $71 \%$ \\
\hline factor $(\mathrm{G}) 12$ & 0,438164 & $<2 \times 10^{-16}$ & Rejeita a Ho & 1,55 & $\begin{array}{c}\text { Aumenta em } \\
55 \%\end{array}$ \\
\hline hora & $-0,115091$ & $<2 \times 10^{-16}$ & Rejeita a Ho & 0,89 & \\
\hline $\begin{array}{l}\text { genda: } \\
\text { - parede A-coberta } \\
\text { - parede A-coberta } \\
\text { - parede A-coberta } \\
\text { - parede A-coberta }\end{array}$ & & $\begin{array}{l}\text { G5 - parede } \\
\text { G6 - parede } \\
\text { G7 - parede } \\
\text { G8 - parede }\end{array}$ & $\begin{array}{l}\text { berta A } \\
\text { berta B } \\
\text { berta C } \\
\text { berta D }\end{array}$ & $\begin{array}{l}\text { G9- } \\
\text { G10-p } \\
\text { G11-p } \\
\text { G12-p }\end{array}$ & $\begin{array}{l}\text {-coberta A } \\
\text {-coberta B } \\
\text {-coberta C } \\
\text {-coberta D }\end{array}$ \\
\hline
\end{tabular}

Fonte: Autor

Pôde-se verificar que o melhor desempenho ocorreu na combinação G8, parede (B) com maior transmitância térmica, combinada com a coberta (D) também com maior transmitância. Esta combinação aumenta a chance da temperatura operativa permanecer abaixo da temperatura base em $169 \%$ em relação ao modelo base. O pior desempenho ocorreu em G11, que representa a combinação da parede (C) e coberta (C), com os maiores valores de atraso térmico. Esta combinação reduziu a chance de ocorrer conforto pela temperatura operativa em $71 \%$, conforme equação abaixo.

$$
\begin{aligned}
& \left(\frac{p}{1-p}\right)=e^{\beta 0+\beta 1 G 2+\beta 2 G 3+\beta 3 G 4+\beta 4 G 5+\beta 5 G 6+\beta 6 G 7+\beta 7 G 8+\beta 8 G 9+\beta 9 G 10+\beta 10 G 11+\beta 11 G 12+\beta 12 \text { Hora }} \\
& e^{0,95+0,79 . G 2+2,04 . G 3+0,50 . G 4+0,60 . G 5+0,44 . G 6+1,18 . G 7+0,37 . G 8+1,43 . G 9+1,19 . G 10+3,48 . G 11+0,64 G 12+1,21 . \text { Hora }}
\end{aligned}
$$

Para diagnosticar as irregularidades do modelo, foram realizadas duas análises de resíduos: 1) o resíduo de Pearson (teste qui-quadrado) que rejeitou a hipótese de que não há diferenças entre a variável resposta e as variáveis independentes, com $p$-value menor que $2,2 \times 10^{-16}$ e ainda, 2) o teste de hipótese de análise de variância (Anova), que rejeitou a hipótese, com resultado de $p$-value menor que $2,2 \times 10^{-16}$. 


\subsection{Discussões}

Nos dois métodos, percebe-se que a influência da coberta é maior que da parede em todas as combinações, em função dos valores de radiação solar na cidade de Patos. A influência da parede é reduzida, mas perceptível nas duas situações.

Ao considerar o método de Graus-hora para Resfriamento, a combinação da parede com alto índice de transmitância térmica associada à coberta com isolamento térmico resultou no melhor resultado. Este evento resulta da possibilidade do isolamento térmico manter condições mais homogêneas no interior da edificação nos horários mais quentes, por reduzir as oscilações térmicas do ambiente, resultando em uma temperatura interna com pequenos intervalos entre o dia e a noite, mas com temperaturas em todos os horários acima do limite de conforto. Nos casos da coberta de fibrocimento sem forro combinada com todas as paredes foram identificados os piores desempenhos térmicos.

Pelo método de Regressão Logística, a coberta de fibrocimento sem forro representa a solução de coberta, dentre aquelas analisadas neste trabalho, com melhor desempenho térmico. Combinada com todas as paredes, a coberta de maior transmitância térmica aumentou a chance de ocorrer conforto no edifício em até $169 \%$.

Pôde-se observar que os dois métodos resultaram em desempenhos inversos, enquanto o método de Graus-hora para resfriamento indica piores condições de conforto no uso de cobertas com maior valor de transmitância térmica, o método de Regressão Logística indica melhores condições de conforto neste tipo de coberta.

As críticas relativas ao método de Graus-hora para Resfriamento reforçam os resultados encontrados nesta pesquisa, pois alguns autores comentam sobre a ausência da distribuição das temperaturas ao longo do ano, permitindo que dois edifícios resultem no mesmo valor de Graus-hora, apesar de um edifício atingir temperaturas bem mais elevadas que outro, como afirma Negreiros (2010).

Foi constatado que no método de Regressão Logística as variações da temperatura não são perceptíveis, assim como no método de percentual de horas de desconforto, pois ambos são métodos que quantificam a chance de ocorrer conforto e desconforto, mas não demonstram este comportamento. O método de Regressão Logística considera quantas vezes a temperatura operativa esteve abaixo da temperatura base, ou seja, na coberta com maior transmitância térmica houve mais ocorrências de temperatura abaixo de $26,5^{\circ} \mathrm{C}$ apesar de não conhecermos o quanto essas temperaturas estiveram acima do limite adotado.

\section{CONSIDERAÇÕES FINAIS}

A escolha adequada do método de avaliação de desempenho térmico é fundamental para a correta interpretação dos resultados. Observou-se que os métodos utilizados são limitados ao representar as condições térmicas encontradas no edifício, resultando na seguinte questão: qual o método mais adequado? Poucas horas de desconforto com grandes oscilações e elevadas temperaturas ou menores oscilações de temperaturas porem maior frequência de temperaturas acima do limite de referência?

A dificuldade em encontrar um método adequado para avaliar o desempenho térmico da envoltória nas condições climáticas escolhidas é uma limitação, pois grande parte dos estudos desenvolvidos nesta área é baseada em definições para climas mais amenos, inclusive os algoritmos utilizados nos programas de simulação computacional, que utilizam referências que não refletem nossa realidade. 
Considerando o uso de modelos numéricos para avaliação de desempenho térmico, recomenda-se a continuidade deste estudo considerando o avanço em relação a algumas limitações encontradas, principalmente no que se refere aos critérios de definição dos métodos de avaliação de desempenho térmico de edificações ventiladas naturalmente.

\section{AGRADECIMENTOS}

Os autores agradecem à CAPES, pelo financiamento da bolsa de mestrado e a professora Dra. Solange Goulart, pelas contribuições e tempo dedicado a esta pesquisa.

\section{REFERÊNCIAS}

ABNT. NBR 15220 - Desempenho térmico de edificações. Parte 3: Zoneamento bioclimático e diretrizes construtivas para habitações unifamiliares de interesse social. Rio de Janeiro. 2005.

ASHRAE. Standard-55P: Termal Environmental Conditions for Human Occupancy. American Society of Heating, Refrigeration and Air Conditioning Engineers, Inc. Atlanta. 2003.

ASTE, N.; ANGELOTTI, A.; BUZZET, M. The influence of the external walls thermal inertia on the energy performance of well insulated buildings. Energy and building, 41 2009. 11811187.

BARRIOS, G. et al. Envelope wall/roof thermal performance parameters for non airconditioned buildings. Energy and Buildings, 50 2012. 120-127.

CARLUCCI, S.; PAGLIANO, L. A review of indices for the long-term evaluation of the general thermal comfort conditions in buildings. Energy and Buildings, 53 2012. 194-205.

CODEIRO, G. M.; ANDRADE, M. G. D. Transformed generalized linear models. Journal of Statistical Planning and interference, V. 139 2009. 2970-2987.

CORDEIRO, G. M.; DEMÉTRIO, C. G. B. Modelos Lineares Generalizados e Extensões. Piracicaba: [s.n.], 2008.

DE DEAR, R. J.; BRAGER, G. S. Thermal comfort in naturally ventilated buildings: revisions to ASHRAE Standard 55. Energy and Buildings, 31 2002. 549-561.

DEVORE, J. L. Probanilidade e estatística: para engeharia e ciências. São Paulo: Pioneira Thomson Learnig, 2006.

GASPARELLA, A. et al. Thermal dynamic transfer properties of the opaque envelope: Analytical and numerical tools for the assessment of the response to summer outdoor conditions. Energy and Buildings, 43 2011. 2509-2517.

GOULART, S. V. G. Dados climáticos para avaliação de desempenho térmico de edificações em Florianópolis. Universidade Federal de Santa Catarina (Dissertação de mestrado). Florianópolis. 1993.

HOSMER, D. W.; LEMESHOW, S. Applied Logistic Regression. $2^{\text {a }}$. ed. New York: John Wiley 7 Sons, 2000.

NEGREIROS, B. D. A. Análise de métodos de predição de conforto térmico de habitação em clima quente-úmido com condicionamento passivo. Universidade Federal do Rio Grande do Norte (Dissertação de mestrado). Natal, p. 115. 2010.

NICOL, J. F.; HUMPHREYS, M. A. Adaptive thermal comfort and sustainable thermal standards for buildings. Energy and buildings, 34 2002. 563-572. 